\title{
Push cone eletrônico de baixo custo para estudo da resistência mecânica ao desenvolvimento radicular das plantas
}

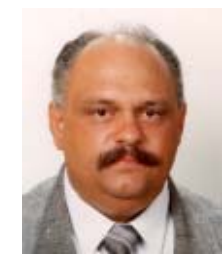

\author{
Nelson L. Cappelli ${ }^{1}$, Claudio K. Umezu² \& Rodrigo F. de Campos ${ }^{3}$ \\ ${ }^{1}$ Laboratório de Instrumentação e Controle - LIC - FEAGRI - UNICAMP, CP 6011, CEP 13083-970, Campinas, SP. \\ Fone: (19) 3788-1052. E-mail: cappelli@agr.unicamp.br (Foto) \\ ${ }^{2}$ Laboratório de Instrumentação e Controle - LIC - FEAGRI - UNICAMP, CP 6011, CEP 13083-970, Campinas, SP. \\ Fone: (19) 3788-1052. E-mail: umezu@agr.unicamp.br \\ ${ }^{3}$ Engenheiro Agrícola. E-mail: e_ferrarini@yahoo.com
}

Protocolo 076 - 29/5/2001

\begin{abstract}
Resumo: Este trabalho descreve a construção, a calibração e os testes de um Push Cone Eletrônico de baixo custo, para utilização em estudos da resistência mecânica à emergência e ao desenvolvimento das raízes das plantas. O equipamento pode ser usado para determinar Índice de Cone até $40 \mathrm{~mm}$ de profundidade. $O$ push cone desenvolvido é constituído de três partes: dispositivo mecânico, circuito eletrônico e software. A parte mecânica é composta, basicamente, de um corpo confeccionado em alumínio, que abriga um cone cuja força para penetração no solo é suprida por uma mola de característica linear; a parte eletrônica é formada por um sensor de deslocamento, utilizado para medir a deflexão da mola, além de um circuito eletrônico, que faz o interfaceamento do sinal do sensor de deslocamento com um microcomputador; o software, tem a função de controlar a aquisição, o armazenamento e o processamento dos dados. 0 equipamento apresentou bons resultados na calibração e nos testes de campo.
\end{abstract}

Palavras-chave: penetrômetro de cone, resistência do solo, resistência a emergência

\section{Low cost electronic push cone for the study of mechanical impedance to root growth}

\begin{abstract}
This work describes the construction, calibration and tests of a Low Cost Electronic Push Cone (LCPC) for the study of mechanical impedance to root growth and plant emergence. The LCPC may be used to determine Cone Index up to a $40 \mathrm{~mm}$ depth. The LCPC developed is made up of three parts: mechanical device, electronic circuit and software. The mechanical part is composed, basically, of an aluminium body that shelters a cone. The force for penetration in the soil is supplied by a spring of linear characteristic. The electronic part consists of a displacement sensor that is used to measure the spring's displacement. An electronic circuit makes the interface of the signal with a microcomputer. The software has the function of controlling the acquisition, storage and processing of the data. The LCPC presented good results in the calibration and in the field tests.
\end{abstract}

Key words: soil cone penetrometer, soil resistance, emergence resistance

\section{INTRODUÇÃO}

Os solos agrícolas estão sujeitos à compactação, devido a fenômenos naturais ou ao tráfego intensivo de máquinas e equipamentos agrícolas utilizados no processo produtivo. Esta compactação restringe a disponibilidade de água e de nutrientes para as culturas e oferece, ainda, resistência mecânica ao desenvolvimento radicular das plantas. Algumas conseqüências da compactação do solo, são: baixo rendimento da cultura e conseqüentes perdas econômicas; diminuição da capacidade de absorção de água no solo, causando escoamento superficial e erosão; arraste de fertilizantes e poluição de mananciais, entre outras.
O estado de compactação dos solos agrícolas, apesar de ser fortemente influenciado pelo teor de umidade do solo, pode ser associado, de forma simplificada, a um índice de resistência à penetração de um cone padronizado, denominado Índice de Cone, obtido por um penetrômetro de cone.

Perumpral (1987) afirma que as principais aplicações dos penetrômetros de cone estão relacionadas: a) à engenharia civil, para obtenção de informações quanto à capacidade de suporte de estaca de fundações; b) à correlação entre o Índice de Cone e os parâmetros de resistência do solo (coesão e ângulo de friç̧ão); c) à predição da capacidade de tração e trafegabilidade de veículos fora-de-estrada; d) à estimativa do estado de compactação do solo; e e) à determinação da resistência 
mecânica do solo ao crescimento radicular e emergência de plantas.

Para a mensuração das camadas de solo compactado através do perfil penetrográfico, existem vários tipos e modelos de penetrômetros e penetrógrafos que foram desenvolvidos ao longo das últimas décadas, como os reportados por Waterways Experiment Station (1948), Carter (1967), Carter (1969), Hendrick (1969), Prather et al. (1970), Wilford et al. (1972), Smith \& Dumas (1978), Phillips \& Perupral (1983), Balastreire \& Amaral (1994) e Cappelli et al. (1995).

O contínuo desenvolvimento dos penetrômetros e penetrógrafos busca minimizar as dificuldades relacionadas, basicamente, ao seu manuseio, à manutenção da velocidade de penetração constante e à aquisição e registro dos dados.

$\mathrm{Na}$ pesquisa do desenvolvimento radicular e emergência das plantas, é comum a determinação do perfil de compactação transversal do solo; para isto, abre-se uma trincheira no solo e se mapeia, com um instrumento denominado "Push Cone", o Índice de Cone na superfície do corte vertical, que abrange a região de desenvolvimento das raízes; este procedimento tem sido feito com um equipamento mecânico, de forma extremamente trabalhosa, anotando-se manualmente cada uma das inúmeras medições e repetições realizadas e, posteriormente, digitadas para o processamento, em uma planilha eletrônica.

Este trabalho objetivou o desenvolvimento de um Push Cone Eletrônico cuja finalidade principal é facilitar o manuseio, a aquisição e o registro dos dados relativos ao Índice de Cone.

\section{MATERIAL E MÉTODOS}

\section{Projeto mecânico}

O Push Cone Eletrônico constitui-se, basicamente, de: corpo cilíndrico usinado em alumínio, cone usinado em aço inoxidável, haste confeccionada com aço carbono e mola helicoidal de compressão linear. O corpo cilíndrico abriga a mola helicoidal que mantém o cone contra seu batente, enquanto a deflexão da mola, que é solidária à haste, ocorre quando o cone é pressionado contra o solo. A Figura 1 mostra o desenho do conjunto mecânico do Push Cone Eletrônico.

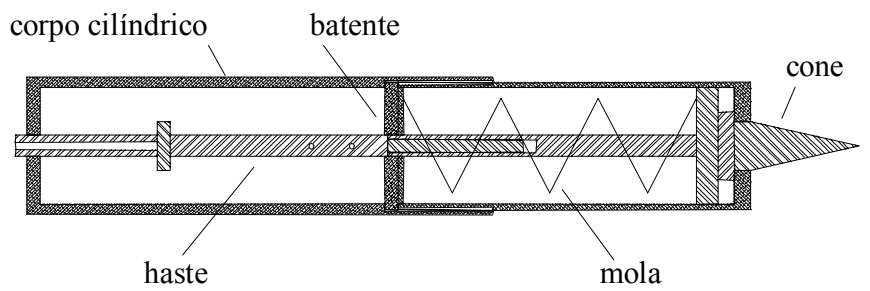

Figura 1. Conjunto mecânico do push cone eletrônico

O cone utilizado possui diâmetro da base de $18 \mathrm{~mm}$ e altura de $40 \mathrm{~mm}$, a mola helicoidal de compressão, especialmente confeccionada em aço carbono, possui diâmetro médio de 32 $\mathrm{mm}$, comprimento livre de $108 \mathrm{~mm}$, diâmetro do arame de $2,5 \mathrm{~mm}$ e constante nominal de $2,0 \mathrm{~N} \mathrm{~mm}^{-1}$, ou seja, nominalmente é capaz de exercer uma força de $80 \mathrm{~N}$ quando deflexionada $40 \mathrm{~mm}$ - compressão máxima devido ao projeto do Push Cone. A força atuante sobre o cone no solo é determinada indiretamente pela medição da deflexão da mola, enquanto o Índice de Cone é determinado pela relação entre a força aplicada e a área da seção do cone correspondente à sua penetração no solo. $\mathrm{O}$ conjunto mecânico pode facilmente ser desmontado, facilitando a limpeza e manutenção do equipamento.

\section{Projeto eletrônico}

Para o funcionamento do Push Cone Eletrônico são necessários a medição e o registro, de forma precisa, da profundidade máxima de penetração do cone padronizado. Um sensor óptico de posição foi especialmente adaptado para realizar tal função. O sensor de deslocamento é constituído por uma fita transparente, com marcas igualmente espaçadas e um conjunto formado por um emissor e um receptor ópticos. A fita é montada solidária à haste e se desloca através do sensor ótico, que é fixado na superfície interna do corpo cilíndrico do equipamento.

O sensor óptico trabalha com fonte de luz do tipo laser (light amplification by stimulated emission of radiation). Uma fonte de luz laser se caracteriza por emitir luz monocromática, coerente e colimada; essas características da luz laser permitem sua utilização em sensores de posição que necessitam de grande resolução, enquanto o sensor óptico utilizado no equipamento é composto de um emissor de luz laser do tipo semicondutor (LED Laser) e por um receptor de luz laser semicondutor (foto diodo laser). O sensor óptico é alimentado por uma tensão de $5 \mathrm{Vcc}$ fornecido por um circuito regulador, e gera um sinal pulsante, cujo número de pulsos é proporcional ao deslocamento da fita óptica.

Um diagrama de blocos do circuito eletrônico do Push Cone está mostrado na Figura 2.

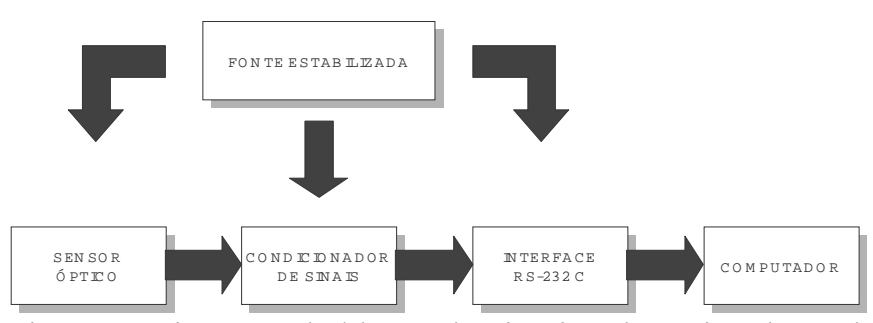

Figura 2. Diagrama de blocos do circuito eletrônico do Push Cone Eletrônico

Um circuito eletrônico foi desenvolvido com a finalidade de alimentar adequadamente o sensor de deslocamento e, ainda, compatibilizar o sinal proveniente de tal sensor com o padrão RS-232C. A alimentação do equipamento é feita por duas baterias de $9 \mathrm{Vcc}$ e apresenta um consumo inferior a $50 \mathrm{~mA}$.

Um interruptor de pressão, montado no corpo do equipamento, é utilizado para iniciar e interromper a coleta de dados. A aquisição dos dados pode ser feita pela interface serial, padrão RS-232C, de um microcomputador. Para operação em campo, recomenda-se o uso de um microcomputador portátil do tipo notebook ou palmtop.

\section{Software}

Para a operação do Push Cone Eletrônico, desenvolveu-se um software dedicado, na linguagem "Visual Basic 3.0", compatível com o sistema operacional Windows. O software possibilita a aquisição, o processamento e o armazenamento 
dos dados e, ainda, permite a visualização dos resultados na tela do microcomputador. Esta última característica permite que os resultados sejam analisados no próprio local do experimento evitando, desta maneira, que eventuais erros sejam notados somente quando os dados forem analisados em laboratório e, em muitos casos, que o experimento seja perdido.

O Push Cone Eletrônico transmite dois sinais através da interface serial, lidos pelo software, em que o primeiro é um sinal de disparo para início de leitura e o outro é sinal pulsante, cujo número de pulsos é proporcional à profundidade de penetração do cone. O software monitora constantemente o sinal de disparo de leitura para, no momento em que o interruptor de pressão for acionado, iniciar a contagem do número de pulsos enviados pelo sensor e finalizar a contagem quando o interruptor de pressão for liberado; desta forma, o software desenvolvido realiza a leitura do número de pulsos, determina a profundidade máxima de penetração do cone no solo e calcula o Índice de Cone, o qual é definido como a relação entre a força aplicada e $\mathrm{a}$ área da base do cone ou área resistente à penetração do cone no solo, e pode ser calculado pela Eq. 1.

$$
\mathrm{IC}=\mathrm{K} \frac{\mathrm{F}}{\mathrm{A}}
$$

donde:

IC - Índice de Cone, $\mathrm{kPa}$

F - Força de penetração, em N

A - Área resistente à penetração, $\mathrm{mm}^{2}$

$\mathrm{K}$ - Fator de conversão de unidades, $1000 \mathrm{kPa} \mathrm{MPa}^{-1}$

A deflexão da mola, após o corpo do Push Cone ter-se encostado ao solo e a força exercida pela mola estar equilibrada com a resistência à penetração do cone no solo, pode ser calculada pela Eq. 2.

$$
\mathrm{d}=\mathrm{K}_{\mathrm{s}} \mathrm{N}_{\mathrm{p}}
$$

em que:

d - Deflexão da mola, mm

$\mathrm{K}_{\mathrm{s}}$ - Constante do sensor óptico, $\mathrm{mm}$ pulso ${ }^{-1}$

$\mathrm{N}_{\mathrm{p}} \quad$ - Número de pulsos, pulsos

A força para a penetração do cone no solo é calculada em função da deflexão da mola, por meio da Eq. 3 .

$$
\mathrm{F}=\mathrm{K}_{\mathrm{m}} \mathrm{d}+\mathrm{P}_{\mathrm{c}}
$$

sendo:

$$
\begin{array}{ll}
\mathrm{F} & \text { - Força exercida pela mola, } \mathrm{N} \\
\mathrm{K}_{\mathrm{m}} & \text { - Constante de proporcionalidade da mola, } \mathrm{N} \mathrm{mm}^{-1} \\
\mathrm{P}_{\mathrm{c}} & \text { - Pré-carga da mola, } \mathrm{N}
\end{array}
$$

A medida em que o cone é introduzido no solo, a área resistente varia e, para o cone utilizado, pode ser calculada pela Eq. 4.

$$
A=0,15904(40-d)^{2}
$$

donde:

A - Área resistente à penetração, $\mathrm{mm}^{2}$

Finalmente, a Eq. 1, que calcula o Índice de Cone, pode ser reescrita de acordo com a Eq. 5.

$$
\mathrm{IC}=\mathrm{K} \frac{\mathrm{K}_{\mathrm{m}} \mathrm{K}_{\mathrm{s}} \mathrm{N}_{\mathrm{p}}+\mathrm{P}_{\mathrm{c}}}{0,15904\left(40-\mathrm{K}_{\mathrm{s}} \mathrm{N}_{\mathrm{p}}\right)^{2}} \quad \text { para } \quad \mathrm{N}_{\mathrm{p}}>0
$$

em que:

$$
\text { IC - Índice de Cone, } \mathrm{kPa}
$$

No software, foram desenvolvidas telas que permitem uma interface gráfica com o usuário. As telas iniciais possibilitam o carregamento de arquivos já existentes ou o salvamento dos dados em formato ASCII (Figuras 3A e 3B) para o processamento posterior dos dados em planilhas de cálculo, softwares estatísticos ou de apresentação gráfica.

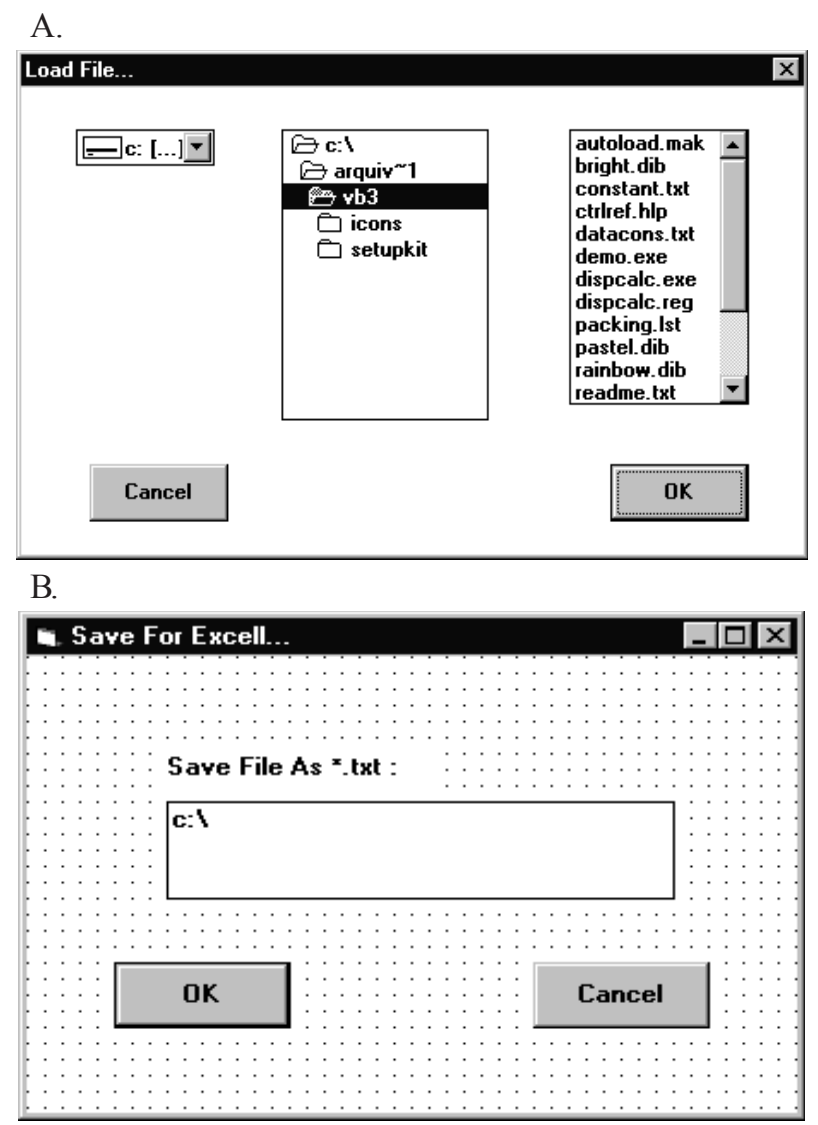

Figura 3. Telas do software: (A) Tela para importação de dados;

(B) Tela para exportação de dados

As telas subseqüentes permitem configurar-se o ensaio, selecionando-se o número de aquisições e sua distribuição espacial (Figura 4A) e acompanhar a realização dos ensaios, visualizando-se instantaneamente o Índice de Cone do ponto selecionado (Figura 4B).

Os dados são transferidos para o microcomputador e armazenados na forma de arquivo ASCII; posteriormente, podem ser processados em uma planilha eletrônica ou em softwares específicos, para cálculos estatísticos ou confecção de gráficos. 
A.

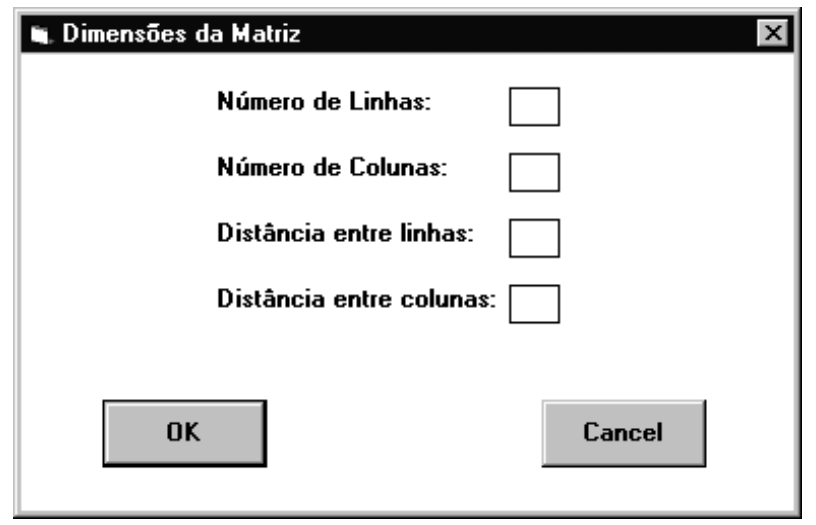

B.

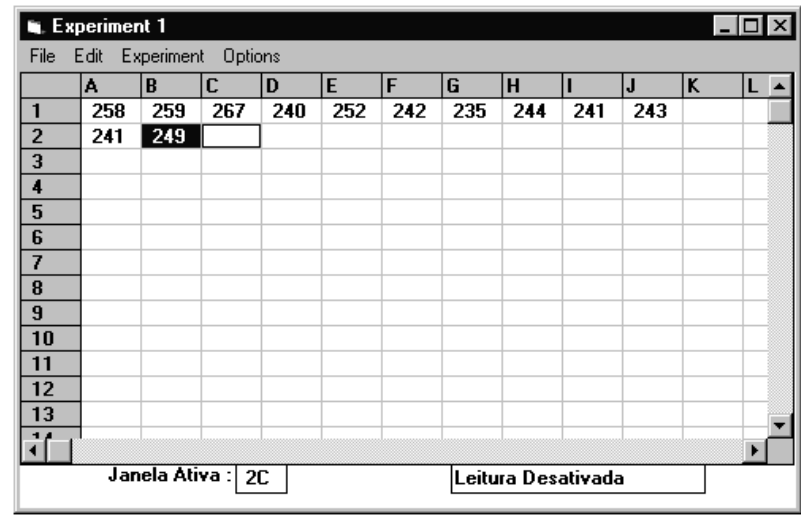

Figura 4. Telas do software: (A) Tela de configuração do ensaio;

(B) Tela de visualização dos dados coletados em tempo real

\section{Teste de campo}

O teste de campo foi realizado abrindo-se uma trincheira no solo e se marcando, numa superfície vertical, uma área retangular com $600 \mathrm{~mm}$ de altura por $1000 \mathrm{~mm}$ de largura. A área foi subdividida em quadrículas de $100 \mathrm{~mm}$, formando uma matriz com 77 pontos amostrais definidos pela intersecção das 7 linhas com as 11 colunas. A Figura 5A mostra a matriz utilizada para o teste do Push Cone Eletrônico em condições de campo. A origem das coordenadas foi tomada no canto superior esquerdo da grade. A Figura 5B indica, em detalhe, a operação de coleta de dados com o Push Cone Eletrônico, cujos dados foram coletados utilizando-se um microcomputador portátil do tipo notebook e, posteriormente, processados em uma planilha eletrônica Excel, na qual foram calculados os respectivos Índices de Cone e confeccionados os gráficos de sua distribuição espacial.

\section{RESULTADOS E DISCUSSÃO}

\section{Calibração}

A calibração do sistema de medição de força do Push Cone Eletrônico, foi realizada fixando-se a estrutura externa e se colocando pesos conhecidos na extremidade do cone. Este procedimento foi realizado para se determinar a relação entre a força aplicada e a deflexão da mola, ou seja, para determinar a constante da mola. Realizou-se uma regressão pelo método dos mínimos quadrados com os dados amostrados e se obteve a Eq. 6 (com um coeficiente de correlação de 0,9993 ), cuja constante $\mathrm{K}_{\mathrm{m}}$ da mola vale $2,004 \mathrm{~N} \mathrm{~mm}^{-1}$, com uma pré-carga na mola de $3,444 \mathrm{~N}$.
A.

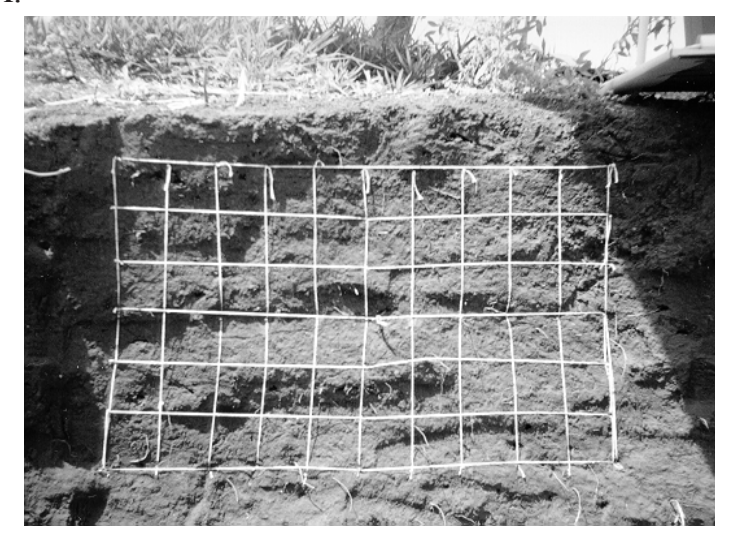

B.

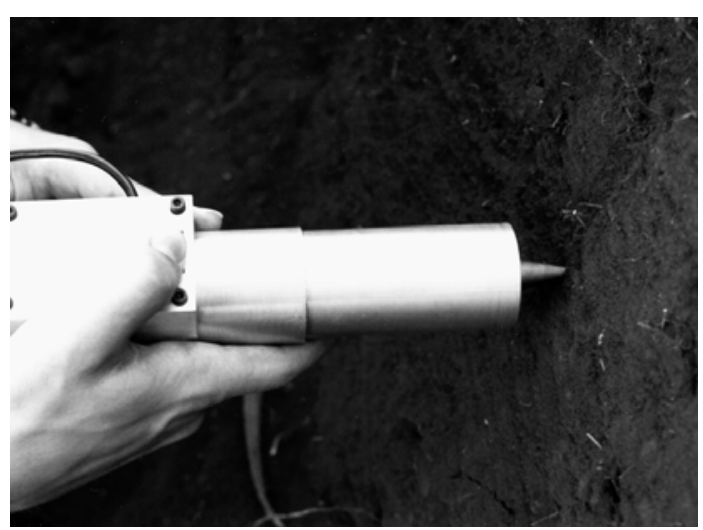

Figura 5. (A) Matriz de pontos do experimento de campo; (B)

Detalhe da utilização do Push Cone Eletrônico

$$
F=2,004 d+3,444
$$

A calibração do sistema de medição da deflexão da mola foi realizada fazendo-se a média de cinco repetições da contagem dos pulsos obtidos para uma deflexão de $40 \mathrm{~mm}$ da mola. A Eq. 2 pode ser reescrita com a constante $\mathrm{K}_{\mathrm{s}}=0,08945$ obtida da calibração, resultando na Eq. 7.

$$
\mathrm{d}=0,08945 \mathrm{~N}_{\mathrm{p}}
$$

Finalmente, a Eq. 5 utilizada para o cálculo do Índice de Cone, pode ser reescrita como:

$$
\mathrm{IC}=\frac{1127,124 \mathrm{~N}_{\mathrm{p}}+21657,445}{\left(40-0,08945 \mathrm{~N}_{\mathrm{p}}\right)^{2}} \text { para } \quad \mathrm{N}_{\mathrm{p}}>0
$$

Os pontos amostrados em um teste de campo, as Isolinhas de Índice de Cone e sua distribuição espacial podem ser observados nas Figuras 6A e B.

O sistema de aquisição de dados via notebook, apresentou o inconveniente de se ter que transportar o microcomputador até o local de medição. Apesar de não representar dificuldade intransponível, está sendo desenvolvido um sistema microprocessado com capacidade de armazenar os dados coletados no próprio Push Cone Eletrônico, o que possibilitará que os dados sejam posteriormente transferidos para um computador. 
A.

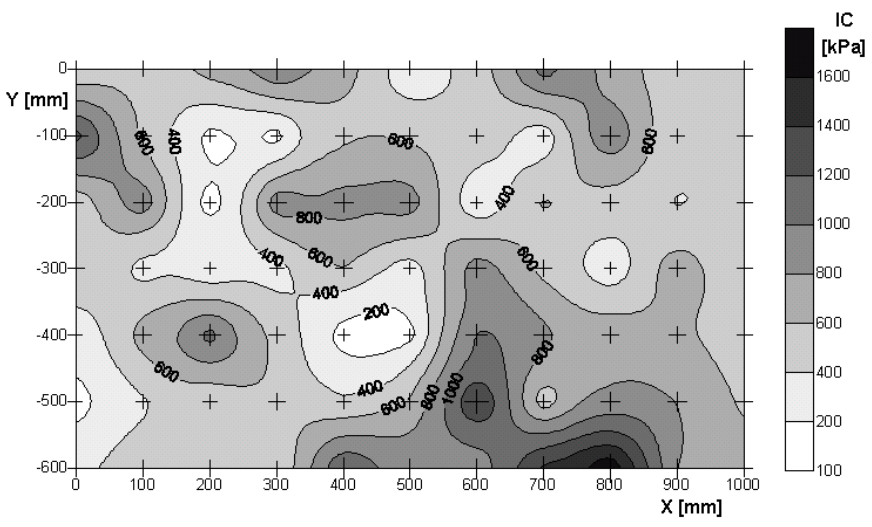

B.

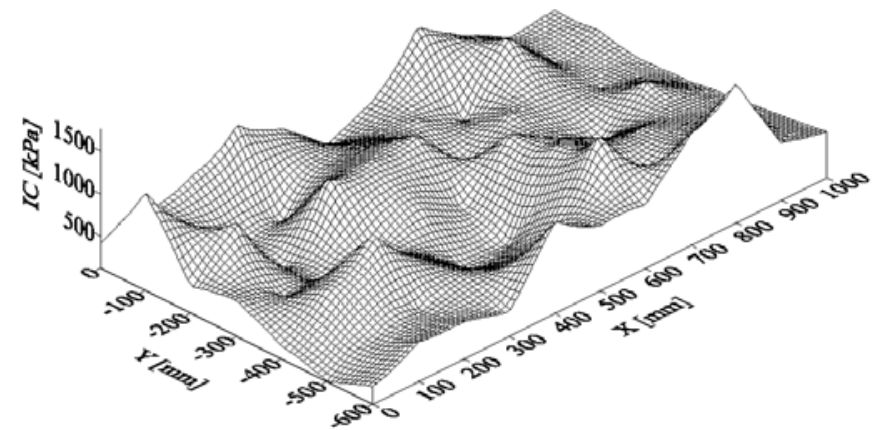

Figura 6. Isolinhas de índice de cone, mostrando sua distribuição espacial e pontos amostrados (A) e distribuição espacial do índice de cone (B)

\section{CONCLUSÕES}

1. O Push Cone Eletrônico desenvolvido alcançou os objetivos do trabalho, pois se mostrou prático em sua utilização e permitiu uma coleta de dados rápida e isenta de erros de operação.

2. O sistema utilizado para a medição da força de penetração, apesar de simples e de baixo custo, mostrou-se adequado, já que a mola apresentou boa linearidade e repetibilidade.

3. O sistema utilizado para medir a deflexão da mola mostrou-se robusto, preciso e adequado para a utilização.
4. O software desenvolvido mostrou-se confiável e de utilização amigável.

\section{LITERATURA CITADA}

Balastreire, L.A.; Amaral, J.R. Desenvolvimento e construção de um penetrógrafo eletrônico. In: Congresso Brasileiro de Engenharia Agrícola, 23, 1994, Campinas. Separatas... Jaboticabal: Sociedade Brasileira de Engenharia Agrícola, 1994. Separata n. 94-4-281.

Cappelli, N.L.; Daniel, L.A.; Miguel, M.A. Penetrômetro eletrônico para determinação do índice de cone de solos agrícolas. In: Congresso Brasileiro de Engenharia Agrícola, 24, 1995, Viçosa. Separatas... Jaboticabal: Sociedade Brasileira de Engenharia Agrícola, 1995. Separata n. 363.

Carter, L.M. Portable recording penetrometer measures soil strength profiles. Agricultural Engineering, St. Joseph, v.46, n.6, p.348, 1967.

Carter, L.M. Integrating penetrometer provides average soil strength. Agricultural Engineering, St. Joseph, v.50, n.10, p.618-619, 1969.

Hendrick, J.G. Recording soil penetrometer. Journal of Agricultural Engineering Research, London, v.14, n.2, p.183-186, 1969.

Perumpral, J.V. Cone penetrometer application - A review. Transaction of the ASAE, St. Joseph, v.30, n.4, p.939-944, 1987.

Phillips, J.; Perumpral, J.V. Designing a microcomputer data logger for a soil cone penetrometer. Agricultural Engineering, St. Joseph, v.64, n.6, p.13-14, 1983.

Prather, O.C.; Hendrick, J.G.; Schafer, R.L. An eletronic handoperated recording penetrometer. Transactions of the ASAE, St. Joseph, v.13, n.1, p.385-386 e 390, 1970.

Smith, L.A.; Dumas, W.T. A recording soil penetrometer Transactions of the ASAE, St. Joseph, v.21, n.1, p.12-14 e 19, 1978.

WES - Waterways Experiment Station. Trafficability as a function of soils - Development of testing instruments. Vicksburg, MS: U.S. Army Corps of Engineers Waterways Experiments Station, 1948. Technical Memo. 3-240 3rd Supplement.

Wilford, J.R.; Wooten, O.B.; Fulgham, F.E. Tractor mounted field penetrometer. Transactions of the ASAE, St. Joseph, v.15, n.2, p.226-227, 1972. 